\title{
Mechanism of Oxygen Reduction Reaction for Sulfur-Doped Graphene Catalyst: First-principles research
}

\author{
Guifa $\mathrm{Li}^{1, \mathrm{a}}$, Hui Wang ${ }^{1}$ \\ ${ }^{1}$ Jiangxi University of Application Science, Institute of Intelligent Manufacturing Engineering,330100, Nanchang, Jiangxi, \\ China
}

\begin{abstract}
The mechanism of oxygen reduction reaction for surfur-doped graphene catalyst was investigated by firstprinciples calculation. Several parameters, such geometry structure, reaction energy and electronic structure were used to analyze the reaction process. The results show that the $\mathrm{OOH}$ molecule absorbed on S-graphene with pyridine structure exhibits chemical adsorption. But the $\mathrm{OOH}$ molecule on S-graphene with pyrrole structure performs physical adsorption. The adsorbed energies of $\mathrm{OOH}$ molecule on S-graphene prove such phenomenon. The electronic structure shows that the excellent ORR of $\mathrm{OOH}$ molecule on S-graphene with pyridine structure comes from their adjacent energy level of valence electron no matter in highest occupied molecular orbital (HOMO) or lowest unoccupied molecular orbital (LUMO).
\end{abstract}

\section{Introduction}

Fuel cell, which can transform the chemical energy straightly into electronic energy, has high power density, quiet and non-pollution[1 3]. However, the oxygen reduction reaction (ORR) is the vital process in fuel cell, air-metal cell and other energy storages applications. Usually, the $\mathrm{Pt} / \mathrm{C}$ is the main catalyst in ORR process. But it has some obvious defects, such as expensive, slow reaction process, low methanol resistance and unstability, which hinders the capability of cell and its commercialization. Then, the substitutes of gold metallic $\mathrm{Pt}$ attract many researchers attention. Exploring metalloid ORR catalyst, especially for doped carbon materials, has been found to a good substitute. Singla et al[4] investigated the sensing performance of grapheme by $\mathrm{Cu} / \mathrm{Pt}$ dual-doping. He found that reactivity of graphene surface for hydrogen adsorption was found to be greatly enhanced with $\mathrm{Pt} @ \mathrm{Cu}$ co-doped graphene surface. Domga et al [5] studied the influence of $\mathrm{N}$ and $\mathrm{P}$ co-doped graphene in ORR under strong alkaline media in advanced chlor-alkali cell. He showed that the $\mathrm{N}$ and $\mathrm{P}$ co-doped graphene showed well exfoliated graphene oxide with few layers of graphene through the various active forms. $\mathrm{Wu}$ et al[6] produced the boron doped graphene for lithium-ion batteries anodes materials. And some elements have been doped in graphene to explore new low-cost catalyst in fuel cell[7 9]. In fact, all of the problems in doped graphene come from the electronic activity on their surface layer. Then in this paper, we research the mechanism of oxygen reduction reaction for

\footnotetext{
${ }^{a}$ Corresponding author: L2877593958@163.com
}

sulfur-doped graphene catalyst by first-principles method.

\section{Simulation models and method}

Reported by Zhang et al[9], two sulfur hybridized graphene sheets $\left(\mathrm{C}_{61} \mathrm{H}_{19} \mathrm{~S}\right.$ and $\left.\mathrm{C}_{60} \mathrm{H}_{18} \mathrm{~S}\right)$ were built with pyridine and pyrrole species as shown in Fig.1, respectively. No matter the carbon or sulfur atoms along the edge of graphene were terminated by hydrogen atoms. A molecular orbital package based on the density functional theory (DFT) is adopted to calculate the oxygen reduction reaction (ORR) process and electronic structures of graphene doping with sulfur. The oxygen reduction reaction processes were simulated firstly with the first electron transmission along with the intermediate molecule $\mathrm{OOH}$ formed. Following with the four-electron pathway presented by Zhang et al[9], the process of $\mathrm{O} 2$ reduced directly to water without involvement of hydrogen peroxide, $\mathrm{O}_{2}+4 \mathrm{H}^{+}+4 \mathrm{e}^{-} \rightarrow 2 \mathrm{H}_{2} \mathrm{O}$, was only simulated in this paper. Then the ORR process of pure graphene and the other less efficient two-step two-electron pathway in which hydrogen peroxide was formed as an intermediate, $\mathrm{O}_{2}+2 \mathrm{H}^{+}+2 \mathrm{e}^{-} \rightarrow \mathrm{H}_{2} \mathrm{O}_{2}$, were not considered anymore. All of these impurity graphene models were relaxed to arrive at its global minimum energy. During optimization and total energy calculation, electronic exchange-correlation energy functions represented in reciprocal space with the Perdew-BurkeErnzerh (PBE) of functional type, which was based on a generalized gradient approximation (GGA), were used [10]. All electrons were included in the calculation. 
Atomic wave functions were developed by double numerical plus d-functions (DND) basis sets [11]. Special K-point method in Monkhorst-Pack scheme [12] was used during the course of Brillouin zone integral. All atomic positions were relaxed according to total energy and force using the BFGS (Broyden, Fletcher, Goldfarb, and Shanno) scheme, based on the geometry optimization criterion ((root-mean-square) RMS force of $0.004 \mathrm{au} / \AA$ and RMS displacement of $0.005 \AA$ ). The calculation of total energy and electronic structure was followed by the geometry optimization with Selfconsistent field (SCF) tolerance of $2 \times 10^{-5} \mathrm{au} /$ atom.
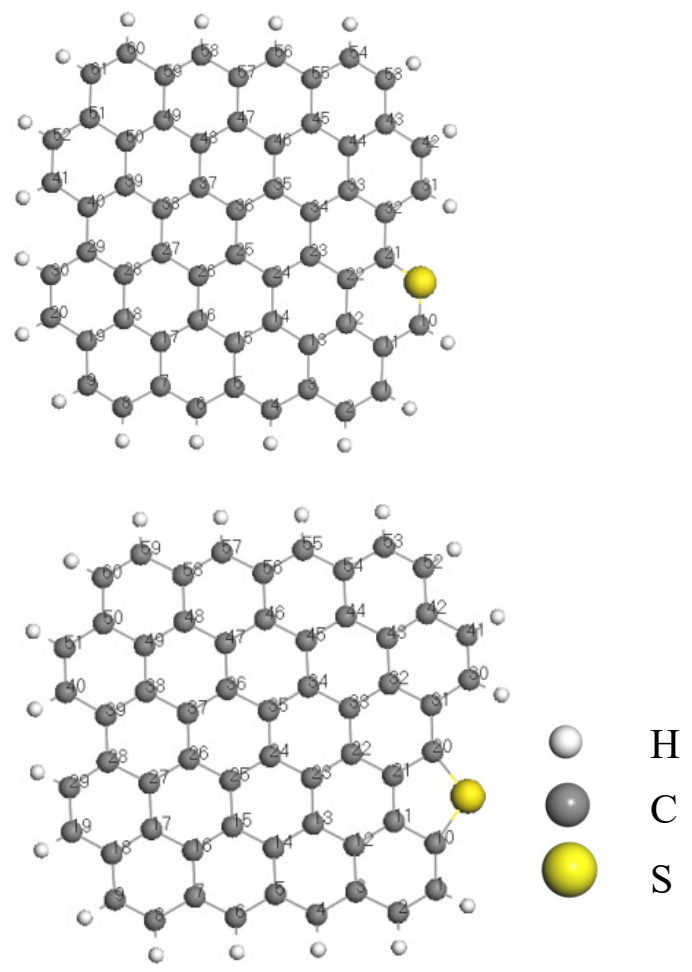

Fig.1. Sulfur-containing graphene sheets of (a) $\mathrm{C}_{61} \mathrm{H}_{19} \mathrm{~S}$ and (b)

$\mathrm{C}_{60} \mathrm{H}_{18} \mathrm{~S}$

\section{Results and discussion}

\subsection{Evolution of geometry structure}

In this paper, we first investigate the ORR behavior of the S-graphene with pyridine structure (Fig. 2(a) (h)). The variation of distance between different atoms is listed in Table 1.

In the first step of the reduction reaction, the $\mathrm{OOH}$ molecule moves from its original state on graphene and absorbs to a carbon atom near sulphur atom. Then the sulphur atom moves out of S-graphene plane to bond with oxygen atom (Fig.2(a)). The distance between the sulphur and oxygen atoms (O1) reduces from $3.30 \AA$ to $1.85 \AA$ (Table1), which the chemical bond is formed spontaneously. This is a vital step for S-graphene to obtain catalytical performance, because atomic absorption and formation of chemical bond are the precondition for the next ORR reaction.
After $\mathrm{OOH}$ absorbed on S-graphene, another $\mathrm{H}$ is introduced to such system. Randomly the $\mathrm{H}$ atom moves to an oxygen atom to form chemical bond. For two type of S-graphene with pyridine and pyrrole structures constructed in this paper, in the next part we will discuss both of their ORR. The ORR process is :

$$
\begin{aligned}
& \mathrm{OOH} \longrightarrow{ }^{*} \mathrm{OOH} \\
& { }^{*} \mathrm{OOH}+\mathrm{H}^{+}+e^{-} \longrightarrow{ }^{*} \mathrm{OH}+{ }^{*} \mathrm{OH} \\
& { }^{*} \mathrm{OH}(\text { absorbed } 1)+\mathrm{H}^{+}+e^{-} \longrightarrow \mathrm{H}_{2} \mathrm{O} \\
& * \mathrm{OH}(\text { absorbed } 2)+\mathrm{H}^{+}+e^{-} \longrightarrow \mathrm{H}_{2} \mathrm{O}
\end{aligned}
$$

wherein aterisk $*$ represents the graphene. This paper began with the reaction (1). Reactions (3) and (4) are the same reaction although the $* \mathrm{OH}$ is absorbed on different carbon atom near sulfur atom. After another $\mathrm{H}$ atom absorbs on $\mathrm{OOH}$ in reaction (2), the distance between two oxygen atoms and $\mathrm{O} 1-\mathrm{G}$ don't change any more, but the distance between the other oxygen atom and graphene reduces to $1.80 \AA$ from primary $2.65 \AA$, which means the $* \mathrm{OH}$ molecule prefers to bond with graphene. When the ORR process occurs at reaction (3), the first $\mathrm{H} 2 \mathrm{O}$ molecule is produced after another $\mathrm{H}$ atom joining. Then the distance between two oxygen atoms increase from $1.39 \AA$ to $2.96 \AA$, which means their chemical bond is broken. And we find the distance of $\mathrm{O} 2-\mathrm{G}$ is also increasing from $3.52 \AA$ to $3.57 \AA$ (Table 1). So the first $\mathrm{H} 2 \mathrm{O}$ molecule is produced and escapes from S-graphene. Then more and more $\mathrm{H}$ atoms absorb on S-graphene, which produce much more $\mathrm{H} 2 \mathrm{O}$ molecule.

About S-graphen with pyrrole structure(Fig. $\left.2\left(a^{\prime}\right) \sim\left(d^{\prime}\right)\right)$, it can be found that its $\mathrm{OOH}$ molecular absorption ability is more hardly than that on the Sgraphe with pyridine. From Table 1, the distance between the oxygen atom and S-graphene is more than $3.0 \AA$ in every reaction steps, which means the $\mathrm{OOH}$ molecule don't absorb on graphene any more. Such result is different from the ORR in $\mathrm{N}$ - graphen[9].

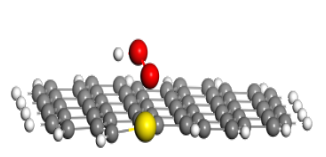

(a) 6G-1

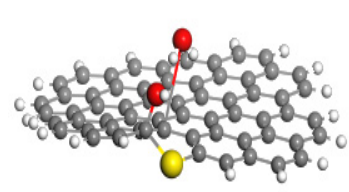

(c) $6 \mathrm{G}-3$

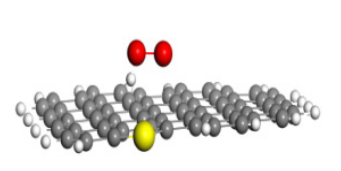

(a’) $5 \mathrm{G}-2$

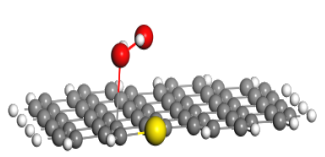

(b) $6 \mathrm{G}-2$

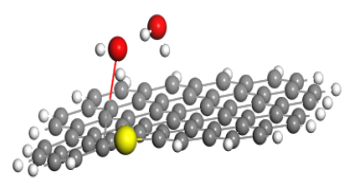

(d) $6 \mathrm{G}-4$

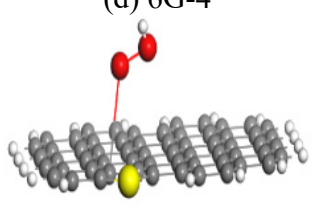

(b’) $5 \mathrm{G}-3$ 


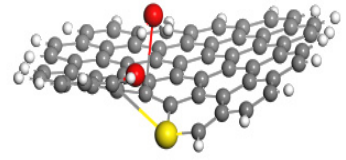

(c') 6G-4
Fig.2 Optimization structures of (a) (d) S-graphene with pyridine and (a') (d') S-graphene with pyrrole

Table 1 Atomic distance during each reaction steps $(\AA)$

\begin{tabular}{cccccc}
\hline & & & & \\
& & Step1 & Step2 & Step3 & Step4 \\
\hline \multirow{2}{*}{ S-Gr } & $d(\mathrm{O} 1-\mathrm{O} 2)$ & 1.41 & 1.39 & 2.96 & 2.88 \\
\cline { 2 - 6 }$($ pyridine $)$ & $d(\mathrm{O} 1-\mathrm{G})$ & 1.85 & 2.76 & 1.45 & 3.72 \\
\cline { 2 - 6 } & $d(\mathrm{O} 2-\mathrm{G})$ & 2.65 & 3.52 & 3.57 & 3.95 \\
\hline \multirow{2}{*}{ S-Gr } & $d(\mathrm{O} 1-\mathrm{O} 2)$ & 1.31 & 1.48 & 3.09 & 2.77 \\
\cline { 2 - 6 }$($ pyrrole $)$ & $d(\mathrm{O} 1-\mathrm{G})$ & 3.10 & 3.50 & 3.37 & 3.43 \\
\cline { 2 - 6 } & $d(\mathrm{O} 2-\mathrm{G})$ & 3.20 & 4.02 & 3.13 & 3.86 \\
\hline
\end{tabular}

\subsection{Reaction energy}

Absorbed energies of molecules on S-graphene are calculated in each reaction process as shown in Fig.3. To be compared obviously, this paper takes the total energy of original S-graphene and $\mathrm{OOH}$ molecules as reference point to be 0 . Then all of the other reaction energies subtract the reference energy to be their relative energy (Fig.3). About S-graphene with pyridine structure (as shown in Figure 3(a)), the relative energy of $\mathrm{OOH}$ molecule absorbed on S-graphene for the first reaction step is equal to $-0.1326 \mathrm{eV}$, which is larger than that ($0.0502 \mathrm{eV}$ ) on S-graphene with pyrrole structure. When another $\mathrm{H}$ atom was introduced into the system, the reaction energies of S-graphene with pyridine structure are equal to $-0.7377 \mathrm{eV},-1.4313 \mathrm{eV}$, and $-2.0575 \mathrm{eV}$ for each reaction steps respectively, wherein the corresponding reaction energies are equal to $-0.6792 \mathrm{eV}$, $-1.9017 \mathrm{eV}$, and $-2.4523 \mathrm{eV}$ for S-graphene with pyrrole (Fig.3(b)).

Conclusively, it can be found that all of the absorbed energies are negative, which means the S-graphene expresses ORR performance. Such results are consistent with the previous part analysis on S-graphene with pyridine structure. But it is opposite with the analysis of S-graphene with pyrrole structure. In Table 1, the oxygen atom doesn't absorb on S-graphene with pyrrole structure anymore because of their large distance. So the S-graphene with pyrrole structure doesn't have any influence on $\mathrm{OOH}$ molecule. Scrutinizing carefully, it isn't hard to find that the decrease of absorbed energy for $\mathrm{S}$-graphene with pyrrole structure is originated from its deformed structure. So the S-graphene with pyrrole in ORR process can't keep its perfect geometry structure and performs unstable property.

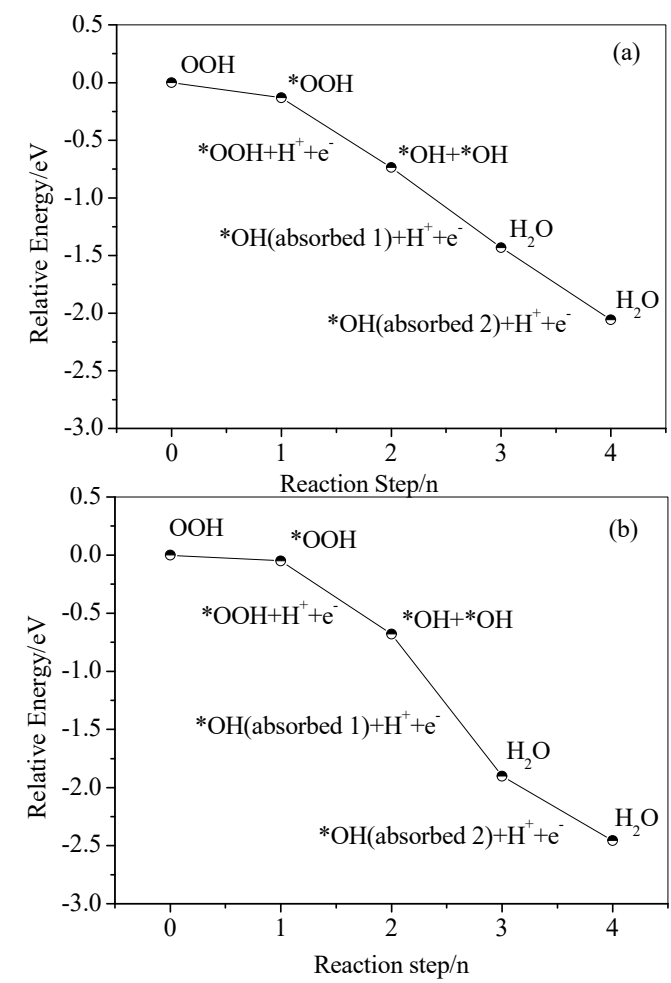

Fig.3 Relative energy of ORR reaction path way on two types of S-graphenes, wherein (a) pyridine and (b) pyrrole structure

\subsection{Electronic density}

In order to discover the influence of their electrons on ORR in different S-graphenes, this paper had calculated their highest occupied molecular orbital (HOMO) and lowest unoccupied molecular orbital (LUMO) along each reaction step. As well known, the $\mathrm{OOH}$ molecule has similar electron level with graphene. Following the reaction rule of quantum chemistry, if the reactants have adjacent energy level of valence electron, their will bond with each other very easily, and the chemical reaction will occur simultaneously. Compared with the HOMO and LUMO of $\mathrm{OOH}$ molecule absorbing on S-graphene with pyridine structure in the first reaction step, their HOMO and LUMO spatial distribution spread homogeneously after $\mathrm{OOH} \rightarrow * \mathrm{OOH}$, which means such molecule state is stable (as shown in Figure 4(a) and (a')). After another $\mathrm{H}$ atom introduced in this system, the electrons of $\mathrm{S}$ atom moves to graphene and $\mathrm{HOOH}$ molecule. It is verified by the morphology of HOMO and LUMO spatial distribution around $\mathrm{HOOH}$ molecule (Figure 4(b) and (b')). Scrutinizing the third and fourth steps of producing $\mathrm{H}_{2} \mathrm{O}$, it is shown that their HOMO and LUMO spatial distribution are similar with each other(Figure 4(c), (c') and Figure 4(d), (d')). So the chemical reactivity of S-graphene with pyridine structure is obviously improved by sulfur doping because of their non-localized HOMO and LUMO distribution. 


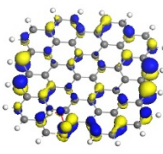

(a) $\mathrm{HOMO}$

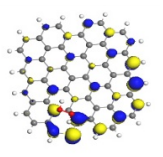

(b)HOMO

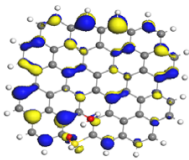

(c) HOMO

(d) HOMO
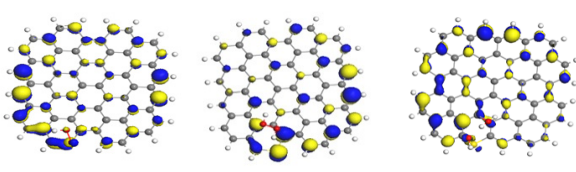

(c')LUMO

(b')LUMO
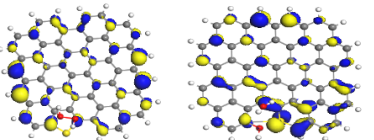

(g) HOMO

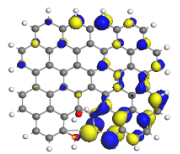

(e)HOMO

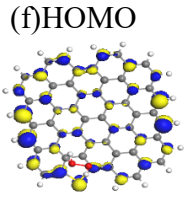

(f') LUMO

(e')LUMO

(g') LUMO

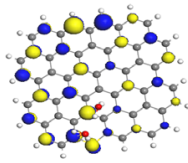

(d')LUMO

(h) HOMO
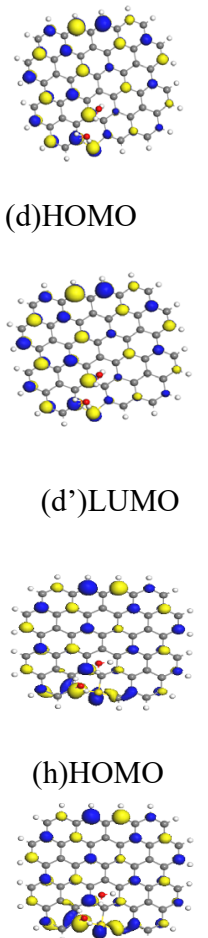

(h') LUMO
Fig.4 HOMO and LUMO spatial distribution of S-graphene with (a) (d) or (a') (d') pyridine and (e) (h) or (e') (h') pyrrole structure in ORR process of Step 1 4.

Focus on the S-graphene with pyrrole structure in ORR process, the LUMO spatial distribution is localized intensively in first step of ORR reaction (as shown in Figure 4(e) and (e')). So the $\mathrm{OOH}$ molecule is hardly absorbed on S-graphene, which is confirmed by previous analysis of geometry structure. In the next three reaction steps, they are similar with each other Figure 4(e $\mathrm{h})$ and $\left.\left(\mathrm{e}^{\prime} \sim \mathrm{h} '\right)\right)$. Their HOMO and LUMO spatial distribution don't have any change in $\mathrm{HOOH} \rightarrow \mathrm{OH}+\mathrm{OH}$ and $\mathrm{OH}+\mathrm{H}$ $\rightarrow \mathrm{H}_{2} \mathrm{O}$ reaction. So the chemical reactivity of Sgraphene with pyrrole structure is not improved by sulfur doping (as shown in Figure 4(h) and (h'))

\section{Conclusion}

Based on the first-principles calculation, the mechanism of oxygen reduction reaction in S-graphene catalysis was researched deeply, the results show that:

1. In the first step of the reduction reaction, the $\mathrm{OOH}$ molecule moves from its original state on graphene and absorbs to a carbon atom near sulphur atom. After $\mathrm{OOH}$ absorbed on S-graphene, another $\mathrm{H}$ is introduced to such system. The distance between the sulphur and oxygen atoms $(\mathrm{O} 1)$ reduces from $3.30 \AA$ to $1.85 \AA$, which the chemical bond is formed spontaneously. About $\mathrm{S}$ graphen with pyrrole structure, it can be found that its $\mathrm{OOH}$ molecular absorption ability is more hardly than that on the S-graphe with pyridine.
2. About S-graphene with pyridine structure, the relative energy of $\mathrm{OOH}$ molecule absorbed on Sgraphene for the first reaction step is equal to $-0.1326 \mathrm{eV}$, which is larger than that $(-0.0502 \mathrm{eV})$ on S-graphene with pyrrole structure. When another $\mathrm{H}$ atom was introduced into the system, the reaction energies of S-graphene with pyridine structure are equal to $-0.7377 \mathrm{eV},-1.4313 \mathrm{eV}$, and $-2.0575 \mathrm{eV}$ for each reaction steps respectively, wherein the corresponding reaction energies are equal to $-0.6792 \mathrm{eV},-1.9017 \mathrm{eV}$, and $-2.4523 \mathrm{eV}$ for S-graphene with pyrrole.

3. Compared with the HOMO and LUMO of OOH molecule absorbing on S-graphene with pyridine structure in the first reaction step, their HOMO and LUMO spatial distribution spread homogeneously after $\mathrm{OOH} \rightarrow * \mathrm{OOH}$. After another $\mathrm{H}$ atom introduced in this system, the electrons of $\mathrm{S}$ atom move to graphene and $\mathrm{HOOH}$ molecule. Focusing on the S-graphene with pyrrole structure in ORR process, the LUMO spatial distribution is localized intensively in first step of ORR reaction

\section{Acknowledgment}

This work was supported by the Innovation special fund project funding of Jiangxi graduate (Grant No.YC2018S357) and Key Laboratory of Jiangxi Province for Persistant Pollutants Control and Resources Recycle (ST201522014).

\section{References}

1. P. Prabhu, V.Jose, J. M. Lee, Matter., 2, 526 (2020).

2. X. Ji, Y. Mu, J. Liang, T. Jiang, J. Zeng, Z. Lin, Y. Lin, J. Yu, Carbon, 176, 21 (2021).

3. J. Theerthagiri, G. Durai, T. Tatarchuk, M. Sumathi, P. Kuppusami, M. Y. Choi, Ionics, 26, 2051 (2020).

4. M. Singla, D. Sharma, N. Jaggi, International J. Hydrogen Eng., 46, 16188 (2021).

5. T. Domga, G. B. Noumi, M. J. Sieliechi, J. B. Tchatchueng, Carbon Trends., 4, 100043 (2021).

6. Z. Y. Wu, , C. Y. Wu, J. G. Duh, Materials Letters 296, 129875 (2021).

7. S. J. Lee, J. Theerthagiri, P. Nithyadharseni, P. Arunachalam, D. Balaji, A. M. Kumar, J. Madhavan, V. Mittal, M. Y. Choi, Renewable and Sustainable Energy Reviews, 143, 110849 (2021).

8. H. Aghamohammadi, N. Hassanzadeh, R. E. Farsani, Ceramics International, 2021, (in press).

9. L. Zhang, Z. Xia, The J. Phys. Chem. C, 115, 11170 (2011).

10. J.P.Perdew, K.Burke, M.Ernzerhof, Phys.Rev.Lett., 77, 3865(1996).

11. Delley, B., J. Chem. Phys., 94, 7245 (1991).

12. J. D. Pack, H. J. Mokhorst, Phys. Rev. B, 16,1748 (1977). 